\title{
CARACTERISTICAS PRODUCTIVAS Y TEXTILES DE LA FIBRA DE ALPACAS DE RAZA HUACAYA
}

\section{A REVIEW OF HUACAYA ALPACAS FIBER TRAITS}

Quispe Peña, Edgar ${ }^{1 *}$, Poma Gutiérrez, Adolfo y Purroy Unanua, Antonio ${ }^{2}$

${ }^{1}$ Programa de Mejora de Camélidos Sudamericanos. PROCASUD. Universidad Nacional de Huancavelica. Perú. ${ }^{2}$ Departamento de Producción Agraria, Universidad Pública de Navarra, Pamplona, España.

*Autor para correspondencia: edgarquispe62@yahoo.com

\section{RESUMEN}

Se realiza una revisión de las principales características productivas (peso de vellón sucio y finura) y tecnológicas (coeficiente de variación de la finura, finura al hilado, índice de curvatura, factor de confor y longitud de mecha) del vellón de la fibra de alpaca, así como de los principales factores medioambientales que afectan dichas características, a fin de demostrar las bondades que tiene la fibra de alpaca para la industria textil, en comparación de las características que se encuentran en otras especies animales. Se llega a la conclusión de que en Huancavelica (Perú) se encuentra un buen potencial para la producción de fibra de alpaca, en cantidad y calidad.

Palabras clave: alpacas, vellón, fibra, características productivas y tecnológicas.

\section{SUMMARY}

A review of the main production (dirty fleece weight and fineness) and technological traits (coefficient of variation of fineness, spinning finennes, curvature index, comfort factor and staple length) of Huacaya alpaca fleece. Also, we attend major environmental factors affecting these characteristics to demonstrate the benefits that alpaca fiber have for the textile 
industry, in comparison of the features in other animal species. We are concluding that in Huancavelica, Peru is a good potential for the production of fiber for quality and quantity.

Key words: alpacas, fleece, fiber, technological and productive traits

\section{INTRODUCCIÓN}

Perú ocupa el primer lugar en el mundo en la producción de alpacas y vicuñas, y el segundo en llamas, después de Bolivia. El aprovechamiento racional de esta ventaja productiva y el uso de la mejora genética de los camélidos (además de vacunos, ovinos y caprinos) es uno de los retos que tiene el país como el medio más efectivo de lucha contra la pobreza y la inseguridad alimentaria, que afecta a numerosas comunidades campesinas que viven de la cría y explotación de estas especies ganaderas.

La industria textil considera a la fibra de alpaca como una fibra especial y, las prendas que se confeccionan con ellas, están clasificadas como artículos de lujo (Wang et al., 2003a). La población mundial de alpacas se estima en unos 3,7 millones (FAO, 2005) y el 80\% de ellas (aproximadamente 3 millones) se encuentran principalmente en las zonas alto andinas de Perú (Puno, Arequipa, Cuzco, Ayacucho, Huancavelica y Apurìmac), de las que alrededor del 86\% son alpacas de color blanco (Brenes et al., 2001); el resto se ubica principalmente en Bolivia y Chile, aunque se han introducido con éxito en Australia, Canadá, Inglaterra, Francia, Nueva Zelanda y Estados Unidos.

El vellón de los camélidos sudamericanos tiene muchas funciones, entre ellos: a) evita la pérdida de agua cutánea, b) protege de las inclemencias climatológicas como la abrasión de la piel, c) permite el camuflaje mediante la coloración, y d) favorece la termorregulación (Grigg et al., 2004), como parte de un mecanismo homeostático relacionado con el metabolismo energético que mantiene al organismo dentro de una rango de temperatura óptima. Referido a esta última función, las fibras permiten a las alpacas una mejor adaptación a las condiciones medioambientales, en particular respecto al aire, pues tiene una mínima conductividad térmica debido que el aire es atrapado en el interior (en la médula) y entre las fibras, resultando un aislamiento inmejorable (Gerken, 2009). En función de éstas características, los componentes del vellón varían con la localización corporal, de este modo es más fino y largo en zonas de la espalda, dorso y flancos, siendo más grueso y corto en zonas de las extremidades y cabeza (Carpio, 1991), debiendo tomarse en cuenta estas 
consideraciones para la determinación de la zona muestral representativa para evaluar las características de la fibra de un vellón de alpaca.

\section{CARACTERISTICAS PRODUCTIVAS DE LA FIBRA DE ALPACAS.}

\section{Peso de vellón sucio (PVS) y diámetro medio de fibra (MDF)}

En el comercio de la fibra, el precio está en función de su cantidad y también de su calidad. Vellones más pesados y de fibras finas valen más que vellones menos pesados y de fibras gruesas, estimándose un coste de producción entre 3,0 y 5,0 dólares americanos, bajo condiciones de cría extensiva (Quispe, 2010). De este modo, el peso del vellón constituye una variable importante que es necesario tener en cuenta en programas de mejora genética de alpacas (Ponzoni et al., 1999; León-Velarde y Guerrero, 2001; Quispe et al., 2009a), así como de ovinos (Villarroel, 1963; Safari et al., 2005), cabras (Kosgey y Okeyo, 2007) y llamas (Wurzinger et al., 2008).

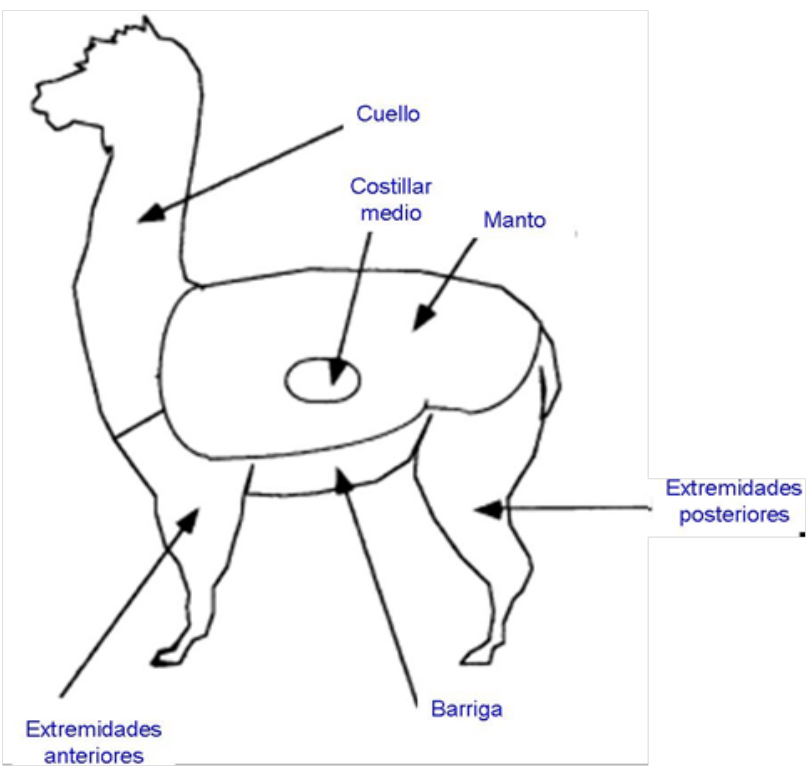

Figura 1. Componentes del vellón de acuerdo a McGregor y Buttler (2004).

En Nueva Zelanda y Australia, Wuliji et al. (2000) y McGregor y Butler (2004) reportaron datos de peso de vellón de alpacas de 2.2 y de 2 a 3.3 kg, respectivamente. Aunque casi se ha generalizado que la fibra de los vellones obtenidos de las alpacas en las 
comunidades campesinas peruanas tiene baja producción y calidad, es posible obtener una producción promedio bianual de $2.30 \mathrm{~kg}$. Sin embargo bajo una cría medianamente tecnificada es posible obtener una producción anual de entre 2.1 a $2.3 \mathrm{~kg}$ (Quispe et al., 2009a; Gutiérrez et al., 2009). Asimismo, Bryant et al. (1989) refieren que el peso de vellón promedio por año para tres niveles tecnológicos, alto, medio y bajo, son del orden de 1.60, 1.40 y $1.20 \mathrm{~kg}$, respectivamente.

La producción de fibra expresada en peso de vellón para un determinado periodo de crecimiento (generalmente de un año) está influenciada por los factores de raza, sexo, localización y, especialmente por la edad de los animales. De modo general se puede considerar que los animales jóvenes producen vellones menos pesados que los adultos (Quispe et al., 2009a). Trabajos realizados en Perú muestran que a la primera esquila (aproximadamente con 10 meses de edad) el vellón de la alpaca pesa $1.15 \mathrm{~kg}$ y aumenta a medida que aumenta la edad del animal, registrándose valores de 1.61, 1.87 y $2.0 \mathrm{~kg}$ a los 2, 3 y 4 años de edad, respectivamente. Mas tarde, los incrementos son mínimos: 2.11 y $2.17 \mathrm{~kg}$ para 5 y 6 años de edad, respectivamente, pero decrece a $2 \mathrm{~kg}$ a los 7 y 8 años de edad (Bustinza, 2001). De igual manera en relación al sexo, se ha encontrado que los vellones de alpacas machos son más pesados que los de alpacas hembras (Castellaro et.al., 1998; Wuliji et al., 2000; Lupton et al., 2006), lo cual se debería al incremento de la superficie corporal (León-Velarde y Guerrero, 2001; Frank et al., 2006; Quispe et al., 2009a), aunque la influencia del sexo podría verse enmascarada por la gestación y la lactación de las hembras que reducirían la producción, como ocurre en las cabras (Newman y Paterson, 1996).

Respecto a la alimentación, Wuliji (1993) indica que tiene un efecto positivo sobre el peso de la fibra de alpaca, lo cual también resulta confirmado por los hallazgos de Newman y Paterson (1994) y Franco et al. (2009), quienes encontraron que el peso del vellón varía con los cambios en la alimentación, tanto en longitud como en diámetro de la fibra, manteniéndose relativamente constante la relación longitud:diámetro. Sin embargo Russel y Redden (1997) encontraron que la relativa contribución de los incrementos en longitud y diámetro parecen ser diferentes, afectando más al peso de vellón sucio el incremento en longitud que el incremento en diámetro.

El diámetro de la fibra es uno de los factores más importantes en la clasificación de la misma, porque determina el precio del vellón en el mercado, a pesar de que la comercialización se realiza por peso del mismo (Villarroel, 1963; Carpio, 1991; Galal, 1986), aunque se otorgan incentivos por finura de vellón. Hasta hace 10 años la medición del 
diámetro de la fibra representaba un problema de coste y de accesibilidad a los métodos tradicionales existentes, especialmente para los pequeños productores (Hoffman y Fowler, 1995). En la actualidad, con el avance de la tecnología y con el impulso que vienen dando los gobiernos en investigación y desarrollo, se constata que los productores alpaqueros tienen mayor accesibilidad para determinar objetivamente la finura de la fibra.

En el ganado ovino desde 1947 existe un método aceptado para evaluar el vellón, respecto a diámetro de fibra, población de fibra, longitud de mecha, densidad de fibra y rizos. La toma de muestra se realiza en la zona del costillar medio [“midside”] (Turner et al., 1953), que se encuentra localizada horizontalmente en la tercera costilla y perpendicularmente en la parte media entre las líneas superior dorsal e inferior ventral. Basado en este método, AylanParker y McGregor (2002) demostraron que en alpacas, la zona del “midside” también resulta representativa para evaluación de la finura media y peso del vellón, constituyéndose por lo tanto en un buen criterio de selección para trabajos de mejora del diámetro de la fibra y del peso de vellón. Algunos investigadores sin embargo utilizan para caracterizar al vellón tres zonas de muestreo: paleta, costillar medio y grupa, lo cual incrementa la mano de obra y los costes de evaluación (Huanca et al., 2007).

En ganado ovino la alimentación tiene un rol importante en la formación y maduración folicular así como en el crecimiento y diámetro de la fibra. Es así que en periodos donde existe poca disponibilidad forrajera, el diámetro de la fibra no solo se reduce, sino también disminuye su crecimiento (Naylor y Hansford, 1999). En alpacas, Russel y Redden (1997) y Franco et al., (2009) reportaron que niveles alimenticios bajos en energía y proteína afinan la fibra, disminuye su crecimiento en longitud y por tanto también disminuye el volumen de la misma (23.97 $\mu \mathrm{m}$ contra $25.75 \mu \mathrm{m}$, $294.7 \mu \mathrm{m} /$ día contra $319.6 \mu \mathrm{m} /$ día y $132.95 \mu \mathrm{m}^{3}$ /día contra $162.79 \mu \mathrm{m}^{3} /$ día respectivamente).

Con respecto al diámetro de la fibra resulta importante el trabajo de Lupton (2006) quien analizó 585 muestras de vellón de alpacas norteamericanas de distintos sexos y edades, encontrando diámetros de fibra de $26.7 \mu \mathrm{m}$ para hembras y $27.1 \mu \mathrm{m}$ para machos; con respecto a la edad, encontró valores de $24.3 \mu \mathrm{m}, 26.5 \mu \mathrm{m}$ y $30.1 \mu \mathrm{m}$ para alpacas de 1 , 2 y 3 ó más años de edad, respectivamente. Por otra parte McGregor (2006) al estudiar alpacas criadas en Australia encontró que el 10\% de alpacas Huacaya presentan una diámetro medio de $24 \mu \mathrm{m}$ y más del 50\% estaban en $29.9 \mu \mathrm{m}$. A su vez, Ponzoni et al. (1999) al analizar un programa de mejora genética para alpacas australianas refiere promedios de diámetro de fibra de $25.7 \mu \mathrm{m}$ con un rango de 23.4 a $27.3 \mu \mathrm{m}$, mientras que Wang et al. (2003a) y Wang et al. 
(2005), también refieren medias de diámetro similares a los encontrados por los anteriores autores.

Entre los trabajos más recientes realizados en alpacas del sur de Perú, destacan los realizados en Arequipa (Renieri et al., 2007; Gutiérrez et al., 2009; Morante et al., 2009; Cervantes et al., 2010), Puno (Apomayta y Gutiérrez, 1998; González et al., 2008; Franco et al., 2009) y Huancavelica (Montes et al., 2008; Oria et al., 2009; Quispe et al., 2009a; Quispe et al., 2009b; Quispe, 2010), que refieren medias de diámetro de fibra desde 21 hasta $24 \mu \mathrm{m}$.

\section{CARACTERISTICAS TEXTILES DE LA FIBRA DE ALPACA}

Muchas son las características que tienen influencia en la transformación de la fibra en tejidos u otros terminales de uso, haciendo que el producto tenga una mayor rentabilidad y sea de preferencia para el consumidor. Las principales características son:

\section{Coeficiente de variación del diámetro de la fibra (CVMDF)}

El coeficiente de variación del diámetro de la fibra (CVDF) es una medida de heterogeneidad del diámetro de las fibras dentro de un vellón y se expresa como el cociente entre la desviación estándar y el promedio multiplicado por 100, por lo tanto su magnitud está expresada en porcentaje. Un vellón con CVMDF más bajo indica una mayor uniformidad de los diámetros de las fibras individuales dentro del vellón (McLennan y Lewer, 2005).

En alpacas, Hack et al. (1999), Aylan-Parker y McGregor (2002), McGregor (2002), McGregor (2006) González et al. (2008), Lupton (2006), Morante et al. (2009), Quispe et al. (2009a) y Quispe (2010) obtuvieron resultados de CVDF de 24.40, 27.00, 23.30, 23.60 18.38, 23.48, 23.12, 22.82 y $21.4 \%$ respectivamente, los cuales, si bien resultan un tanto elevados, muestran una alta variabilidad de los animales que resulta conveniente para programas de mejora genética. Asimismo, casi todos los resultados (a excepción de lo encontrado por Aylan-Parker y McGregor (2002), no superan el 24\%, que representa el límite para rendimientos textiles acorde a su diámetro, y que se encuentra asociado al rendimiento del hilado, propiedad conocida también como finura al hilado (Quispe et al., 2009a).

\section{Factor de confort (FC) y factor de picazón (FP).}

El factor de confort (FC) se define como el porcentaje de las fibras menores de $30 \mu \mathrm{m}$ que tiene un vellón y se conoce también como factor de comodidad. Si más del 5\% de fibras son mayores a $30 \mu \mathrm{m}$, entonces el tejido resulta ser no confortable para su uso por la picazón 
que siente el consumidor en la piel (McLennan y Lewer, 2005). Contrariamente, el porcentaje de fibras mayores a 30 micrones se conoce como el factor de picazón (FP). Por tanto, la industria textil de prendas prefiere vellones con un FC igual o mayor a 95\% con un FP igual o menor a 5\%. Estos dos parámetros valoran los intercambios de sensaciones entre el cuerpo humano y la prenda de fibra ante las respuestas fisiológicas y sensoriales de las personas (Sacchero, 2008).

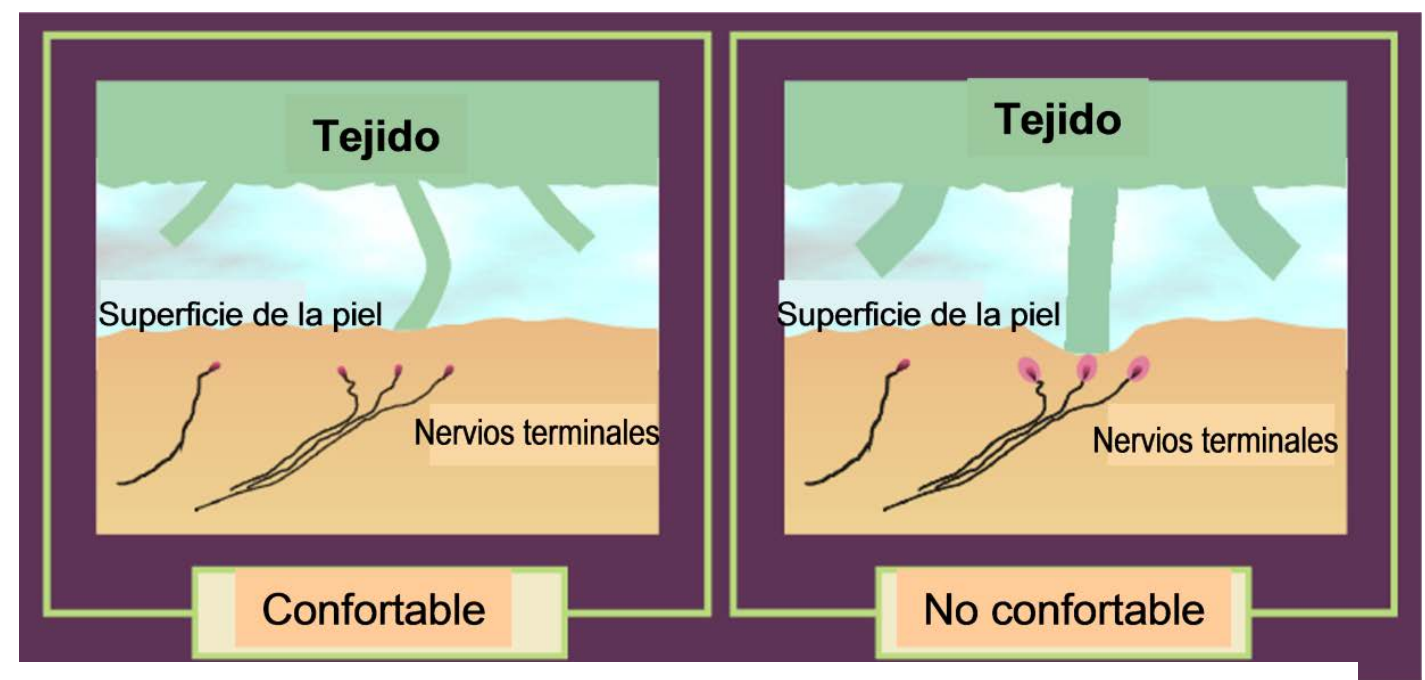

Figura 4. Interacción entre el tejido, los terminales de la fibra o lana y la piel, que muestran la importancia del factor de confort. (Modificado de: Garnsworthy et al., 1988).

Durante el uso de las prendas, los terminales de la fibra emergen hacia la superficie y presionan contra la piel. La fuerza que el terminal de la fibra puede ejercer sobre la piel antes de flexionarse es altamente dependiente de su diámetro y longitud de emergencia. Por encima de la fuerza crítica (100 mg) los nervios que se encuentran situados justo debajo de la piel son provocados. Cuando se reciben muchas de estas señales el cerebro lo interpreta como una sensación no placentera, comúnmente llamada picazón. Para un tejido plano usado comúnmente en chompas o suéteres, el diámetro crítico que conlleva a la picazón es aproximadamente de 30 a $32 \mu \mathrm{m}$, aunque esto varía considerablemente entre personas, temperatura y limpieza de la piel. En prendas normales confeccionadas con lana que exhiben una media de $21 \mu \mathrm{m}$ tienen un número pequeño de fibras con diámetros mayores a $30 \mu \mathrm{m}$, lo que le da confortabilidad a la prenda (Naylor y Stanton, 1997).

El factor de picazón no es un carácter técnico de la fibra, sino que más bien está relacionado con el grado de confort que brindan las prendas fabricadas con fibra de alpaca 
sobre el usuario (Sacchero (2005). McGregor y Butler (2004) obtuvieron en alpacas criadas en Australia, un factor de picazón de 44.42 \% y un índice de confort de 55.58 \%. Ponzoni et al., (1999), en un estudio realizado en alpacas al sur de Australia, muestran un índice de confort de 75.49 \%, mientras que Lupton et al. (2006), en alpacas Huacaya criadas en EEUU y con una muestra representativa de 585 animales, hallaron un índice de confort de $68.39 \pm$ $25.05 \%$.

Quispe et al., (2009a) en alpacas de color blanco provenientes de 8 comunidades de la región de Huancavelica (Perú), de distintas edades y sexos, encontraron valores de factor de picazón de 6,33\% $\pm 0,30 \%$ que correspondería a un factor de confort de 93,67\%, el cual se considera como un buen factor acorde a los requerimientos de la industria textil. Se sabe que mientras las fibras tienen menor diámetro el confort es mayor. Asimismo, Quispe (2010) reportó una suficiente evidencia del efecto de la edad, año y comunidad sobre ésta característica.

\section{Índice de curvatura.}

El índice de curvatura (IC) de la fibra es una característica textil adicional que puede ser utilizado para describir la propiedad espacial de una masa de fibras de lana. Esta propiedad, que es común a todas las fibras textiles, es de interés para los fabricantes de alfombras y prendas de vestir. Los fabricantes de fibras sintéticas introducen rizos a sus fibras y filamentos a fin de mejorar la densidad de sus productos textiles (Fish et al., 1999). El rizado de la lana, expresado como curvatura de fibras, se puede medir utilizando los equipos como la OFDA (Analizador óptico del diámetro de fibras) y LaserScan, ambos de fabricación australiana (Quispe et al., 2008a).

El rizo de la fibra, medido objetivamente mediante el IC, es una característica deseable respecto al tacto, aunque a veces también puede crear dificultades en referencia al procesamiento. El rizo en una mecha de lana puede ser expresado en función a la "definición del rizo”, descrita como el grado de alineamiento del rizo, de modo que lanas donde el rizo de la fibra no se encuentra bien alineado tienen definiciones pobres, y a la "frecuencia del rizo” definido como el número de longitudes de ondas curvadas por centímetro. Ambas características, junto con el color de la grasa, la longitud de mecha, la suciedad y el desgaste representan el "estilo de la lana”, el cual es muy importante para determinar el rendimiento al procesamiento, prácticas de comercialización y calidad de los productos de lana final. 
La curvatura de las fibras puede ser en tres dimensiones, debido a que las fibras se encuentran flexionadas y torcidas a lo largo de su longitud. Sin embargo, debido a que la mayor parte de la curvatura ocurre en un plano y teniendo la flexión la mayor contribución, la forma de la fibra puede ser representada en una forma de onda bidimensional (Fish et al., 1999).

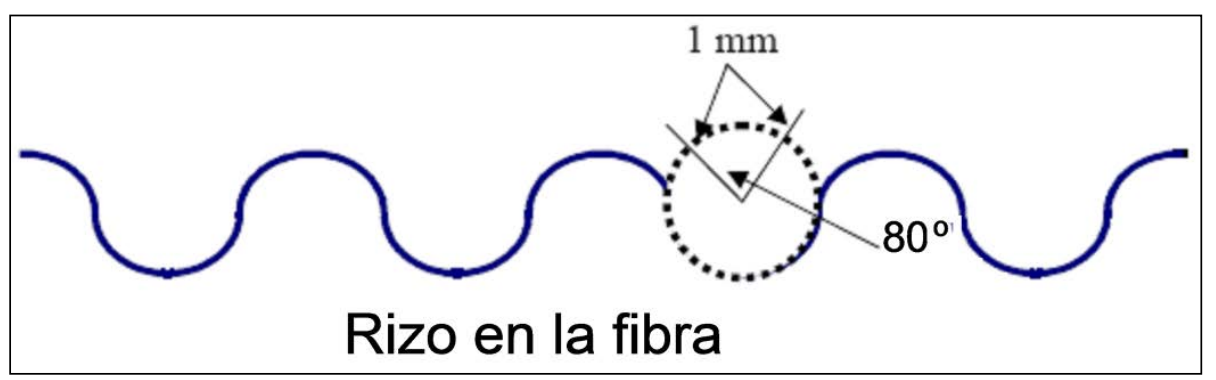

Figura 2. Representación bidimensional de la forma de una fibra de lana. (Tomado de Fish et al., 1999).

Existen relaciones directas entre el IC de la fibra con la frecuencia de rizos en la mecha y con la resistencia a la compresión (los coeficientes de correlación varían entre 0.8 y 0.9). También existe una fuerte relación entre la media del diámetro de fibra y la curvatura de la fibra, donde fibras con alta curvatura tienen fibras con menor diámetro (Fish et al., 1999). Holt (2006) reportó coeficientes de correlación entre el índice de curvatura [expresado en grados / milímetro $\left({ }^{\circ} / \mathrm{mm}\right)$ ] y el diámetro de fibra (expresado en $\left.\mu \mathrm{m}\right)$ de 0.64 y 0.79 para muestras de fibra de alpacas Huacaya y Suri, y entre frecuencia de rizo y diámetro de 0.44, demostrando la ventaja que tiene el índice de curvatura frente a la frecuencia de rizos, cuando se quiere evaluar el diámetro de la fibra.

Muchos estudios han sido dedicados a evaluar el efecto del rizo de la mecha de fibra sobre el rendimiento al procesamiento y la calidad de los productos lanares. Hansford (1996) reportó que lanas con baja frecuencia de curvaturas o rizos y alta definición de rizo conlleva a obtener una longitud media de fibra (Hauter) más larga en los “tops” (cinta de fibra obtenida después del peinado). Para las lanas superfinas, una menor frecuencia de rizos en la fibra da lugar a una mayor uniformidad de hilados y menor número de terminales salientes en la hilatura (Wang et al., 2004). 
El IC en alpacas ha sido estudiado en Perú por Siguayo y Aliaga (2010), quienes encuentran valores entre 47.66 y $54.01 \% / \mathrm{mm}$ en alpacas, mientras que Quispe (2010) encuentra una media de 38.8 \% $\mathrm{mm}$. Así también, el IC está bien documentado en países como Australia, Nueva Zelanda y Estados Unidos principalmente, basta referir a los resultados de Liu et al. (2004), Wang et al. (2004), Lupton et al. (2006), McGregor (2006) quienes encontraron valores de 28.0, 32.0, 32.5, 32.2 y 27.8 \% $/ \mathrm{mm}$, respectivamente. Al parecer, la fibra de alpaca Suri tiene menor curvatura que la Huacaya $\{15$ a 35 contra 25 a 60 \% $\mathrm{mm}$ respectivamente (Holt, 2006)\}, mientras que la lana de ovino tiene mayor índice de curvatura que la fibra de alpaca (Liu et al. 2004; Wang et al. 2004), pero menor que la de vicuña (Quispe et al., 2010).

\section{Finura al hilado}

La finura al hilado (FH) expresada en $\mu \mathrm{m}$ (spinning fineness), provee una estimacion del rendimiento de la muestra cuando es hilada y convertida en hilo. Su estimación proviene de la combinación de la media del diámetro de fibra (MDF) y el coeficiente de variación (CVMDF). La idea original viene de Martindale (1945), que fue analizada y planteada por Anderson (1976) como “effective fineness” y que, posteriormente fue modificada por una ecuación práctica llamándose a dicho valor finura al hilado (Butler y Dolling (1995) y es una característica fuertemente heredable (Butler y Dolling, 1992). La ecuación se normaliza bajo un coeficiente de variación del $24 \%$ en la cual la finura al hilado es lo mismo que la media del diámetro de fibra previa al procesamiento (Lupton et al., 2006).

Anderson (1976) llamó a la expresión: $F_{e}=M D F^{*} \sqrt{1+5^{*}(C V M D F / 100)^{2}}$, finura efectiva (effective fineness) y lo usó con la finalidad de demostrar la influencia de los cambios de la MDF y el CVMDF sobre la uniformidad de los hilados. Dos tops con diferentes MDF y CVMDF pueden producir hilados de la misma uniformidad, si sus finuras efectivas tienen el mismo valor al utilizar la fórmula anteriormente descrita. Por ejemplo, un top con MDF y CVMDF de $21.5 \mu \mathrm{m}$ y $20.0 \%$ respectivamente, produce un hilado más uniforme que otro top con MDF y CVMDF de 20.2 y 27\% respectivamente (De Groot, 1995).

La finura efectiva sólo depende de la MDF y del CVMDF y es siempre numéricamente mayor que la MDF, aunque esto puede corregirse normalizando la finura efectiva mediante la aplicación de la ecuación: $F \approx 0.881 * M D F * \sqrt{1+5 *(C V M D F / 100)^{2}}$ (Butler y Dolling, 1995). 
En alpacas Huacaya de color blanco, Quispe (2010) encontró una finura al hilado de $20.9 \mu \mathrm{m}$ observando que animales jóvenes tienen menor FH que animales adultos y que los animales menores de 18 meses son los que exhiben una mejor FH; asimismo, encontró efectos altamente significativos de factores como año y comunidad, sobre dicha finura.

\section{Otras características que determinan la calidad de la fibra.}

Debido a que la calidad de la fibra es una función compleja (Wang et al. 2003b), muchas son las características que están involucradas en ella, siendo las principales:

\section{- Punto de rotura.}

El punto de rotura es la información que se desprende de la determinación de la resistencia a la tracción, e indica el porcentaje de mechas que se rompen en la punta, mitad y base de la mecha. Está muy relacionado con la longitud media de las fibras que conforman el top (hauteur). La rotura de fibras está relacionada con los lugares de menor diámetro en el perfil de la fibra, producidos por factores nutricionales, ambientales, sanitarios, etc. Desde el punto de vista del industrial transformador la peor situación se da cuando la mayoría de las mechas se rompen por la mitad, porque reduce el valor del hauteur, especialmente cuando la resistencia a la tracción es baja (Sachero, 2005).

\section{- Resistencia a la tracción}

Durante el procesamiento textil que involucra el lavado, cardado, peinado, teñido, mezclado entre otros, las lanas y fibras son sometidas a diversas tracciones que pueden lograr romper la fibra (McGregor, 2006).

La resistencia a la tracción puede ser descrita como la fuerza de tensión requerida para romper una cantidad de lana o fibra conocida. Tanto el tipo de muestra como el calibre de las fibras tienen influencia en los resultados. La medición de la resistencia a la tracción intrínseca de la fibra es evaluada en una sola fibra, sin embargo, esto resulta tedioso y solo sirve como técnica de investigación. Un método más eficiente viene a ser la prueba de resistencia que es medida en un haz de fibras. Los resultados están en función de la calidad intrínseca de la fibra más que en las variaciones en el diámetro (Hansford, 1996).

La resistencia a la tracción se mide en Newton/kilotex, donde el Newton (N) es una fuerza o carga y el kilotex es la densidad lineal de una mecha o un sliver ( $\mathrm{gr} / \mathrm{cm})$. En lanas puede variar desde niveles muy bajos hasta 90 N/ktex. Lanas con valores mayores a 
$50 \mathrm{~N} / \mathrm{ktex}$ son consideradas muy resistentes, entre 50 a $30 \mathrm{~N} / \mathrm{ktex}$ resistentes, con valores menores a $30 \mathrm{~N} / \mathrm{ktex}$ son consideradas débiles y con menos de $15 \mathrm{~N} / \mathrm{ktex}$ quebradizas (Sachero, 2005). Una resistencia mayor a $30 \mathrm{~N} / \mathrm{ktex}$ es considerada adecuada para la industria textil, y las fibras de alpaca son bastante fuertes, pues exhiben una resistencia a la tracción de alrededor de 50 N/ktex (Lupton et al., 2006). Tanto Wuliji et al., (2000) en Nueva Zelanda y McGregor (2006) en Australia han encontrado valores en fibra de alpacas adecuado para el procesamiento de 31.8 y 76.0 N/ktex, respectivamente.

Cambios medioambientales pueden repercutir en una reducción del diámetro de fibra, que puede conducir a la reducción en la resistencia a la tracción (Naylor y Stanton, 1997; Quispe et al., 2008b; Poma et al., 2009; Mayhua et al., 2011). Asimismo, factores intrínsecos pueden tener efecto sobre ésta variable, tal es el caso del sexo y el color, pues los machos tienen fibras mas resistentes que las hembras (38.7 contra $33.4 \mathrm{~N} / \mathrm{ktex}$ ) y las fibras claras son más resistentes que las oscuras (45.6 contra 39.2 N/ktex) (Lupton et al., 2006)].

\section{- Resistencia a la compresión}

La resistencia a la compresión (RtC) es una característica objetiva que refleja la compresibilidad de la fibra (Wang et al., 2003a; Liu et al., 2004) y es medida en kilopascales $(\mathrm{kPa})$. Un pascal es una unidad de presión equivalente a un Newton por $\mathrm{m}^{2}$. En el sector comercial, valores de RtC mayores a $11 \mathrm{kPa}$ son considerados altos, de $8 \mathrm{a}$ $11 \mathrm{kPa}$ medios y menores a $8 \mathrm{kPa}$ bajos. La fibra de alpaca tiene una RtC baja debido a que tiene bajos niveles de rizo. De este modo, la fibra la alpaca no es apta para la confección de prendas o artículos que requieran tener gran volumen o alta resistencia a la compresión (Lupton et al. 2006).

La fibra de alpaca Huacaya muestra valores de RtC entre 4,5 a 5,4 kPa (Wang et al., 2003a; McGregor, 2006) mientras que para la lana de ovino el rango es de 5 a 15 kPa, existiendo en alpacas una relación directa entre la RtC y la media del diámetro de la fibra $\left(\mathrm{R}^{2}=0,16\right)$. De este modo fibras mas gruesas muestran RtC más altos, lo cual resulta contrario a la lana de ovino, donde existe una relación inversa $\left(\mathrm{R}^{2}=0,55\right)$. Los bajos valores de RtC que se encuentran en la fibra de alpaca podrían deberse a la baja altura de las células de la cutícula $(9,5 \mu \mathrm{m})$, mayor frecuencia de rizos $(10,5 / 100 \mu \mathrm{m})$ y a la delgadez de las cutículas (375nm), pues en la lana de ovino se encuentran valores alrededor de $13,2,7,6 / 100 \mu \mathrm{m}$ y $1098 \mathrm{~nm}$ respectivamente para cada característica 
indicada anteriormente (Liu et al., 2004). La delgadez y la mayor frecuencia de células cuticulares en la fibra de alpaca reducirían la resistencia a la fricción cuando las fibras son comprimidas, y por tanto resultarían los valores reducidos de RtC en alpacas.

$\mathrm{Al}$ parecer el sexo y edad no tienen efecto sobre la RtC, pero el color y el año influirían fuertemente sobre esta variable, observándose que colores más oscuros tienen menor RtC que colores claros (Lupton et al., 2006).

\section{- Tasa de medulación.}

Algunas fibras de alpaca aparte de la cutícula y corteza, tienen una médula en la parte media que se extiende a lo largo de la fibra (Rui-wen et al., 2008), y tiene un efecto aislante, de modo que las prendas que son confeccionadas con dichas fibras mantienen calor en quienes los usan (Wang et al., 2005). Sin embargo para fines del procesamiento la presencia de la médula supone un problema importante, especialmente en el teñido porque causa una mayor refracción de la luz que hace aparecer las fibras teñidas más claras (Rodríguez, 2006)

En llamas, Martínez et al. (1997) encontraron tasas de medulación de 20.2, 37.7 y 39.4\% para fibras no meduladas, fibras con medulas fragmentadas y fibras con médula continua, mientras que Contreras y Quispe (2010) en alpacas Huacaya de color blanco, encontraron una tasa de medulación de $66.49 \%$ y, respecto al tipo de medulación, reportan tasas de medulación de 33.51, 16.14, 20.38, 12.74 y $17.24 \%$ para fibras sin médula, con médula poco continua, médula muy continua, médula continua larga y médula continua, respectivamente.

\section{- Rendimiento al lavado}

El rendimiento al lavado es la proporción de lana o fibra que queda de una muestra una vez lavada. Para dicho proceso las muestras son colocadas en máquinas lavadoras que operan con los mismos principios que los lavaderos comerciales. El lavado se realiza con detergente en agua caliente, después son enjuagados dos veces con agua fría. En este proceso se elimina todo el "suint” (cera) y cerca del 98 \% del contenido solido (tierra y suciedad) y grasa. Una malla fina en la base de los lavaderos retiene la lana y toda la materia vegetal, que son colocadas en cápsulas especiales para el secado. Las cápsulas se centrifugan, para eliminar el agua excedente. Después del lavado y del secado tres tipos de contaminantes continúan en la lana: toda la materia vegetal, pequeñas cantidades de 
grasa residual y algo de suciedad. Para determinar la Base Lana, estos tres contaminantes necesitan ser cuantificados (Sachero, 2005).

Cuando se lavan las fibras de alpaca, pierden poco peso debido a que tienen poca cantidad de polvo, materia vegetal, cera y grasa en comparación con la lana de ovino. De este modo los vellones de alpaca tienen un rendimiento al lavado entre 75 y 82\% de fibra, mientras lana de oveja Merino tiene 49 \%. Wuliji et al. (2000), Aylan-Parker y McGregor (2002) McGregor (2006) y Lupton et al. (2006), encontraron valores entre 89 a 95 \% para las alpacas, mientras que en la lana de ovino el rendimiento encontrado fue entre 59 y 77 \% para diferentes razas (Mueller, 1991).

\section{FACTORES QUE AFECTAN A LA CALIDAD Y A LA CANTIDAD DE FIBRA.}

Los factores que influyen en la cantidad y la calidad de la producción de fibra en camélidos sudamericanos se clasifican en factores medioambientales externos y factores genéticos o internos. Los factores externos que modifican la respuesta productiva en alpacas son la alimentación (Russel y Redden, 1997), la locación geográfica o lugar de pastoreo (Quispe et al., 2009a) y, en el caso del peso de vellón, es particularmente relevante considerar la frecuencia, año de esquila (Ruiz de Castilla, 2004) y la precipitación pluvial (Bustinza, 2001). La altitud no ejerce influencia ni sobre la cantidad ni la calidad de fibra (Braga et al., 2007). Entre los factores internos que afectan el diámetro de fibra y peso de vellón resaltan el sexo, la edad (Quispe et al., 2009a), la raza (Cervantes et al., 2010), sanidad, estado fisiológico (Franco y San Martín, 2007), condición corporal (Carhuapoma et al., 2009.) y color de vellón (McGregor y Butler, 2004; Renieri et al., 2007; Oria et al., 2009).

El peso del vellón sucio de una alpaca, es función del número de fibras y del peso medio de estas fibras. La producción de fibra depende predominantemente del funcionamiento de los folículos pilosos, en periodos largos de crecimiento, pues la fase anágena (con una zona matricial en mitosis) es la que predomina (Rogers, 2006), frente a la fase catágena (donde se detiene el crecimiento folicular por estrechamiento del bulbo, reducción de la papila pilosa y arrugamiento del saco piloso y queratinización) y telógena (donde se reduce de tamaño el folículo por reducción de la papila, matriz y saco piloso) (Torres de Jasaui et al., 2007). Contrariamente, en ratones, conejos y cobayos el motor folicular progresa a través de estados de crecimiento, regresión y regeneración en forma de ondas a través del cuerpo (Rogers, 2006). 
La actividad de los folículos se ve influida por diversos factores ambientales y fisiológicos. El más importante de los factores ambientales es la cantidad y calidad de nutrientes que llegan a los folículos, aunque también pueden influir sobre la producción de los folículos distintos factores fisiológicos como la gestación y la lactación, el sexo, la edad, la sanidad y el clima. Todos estos factores actúan sobre la producción de fibra diaria de los folículos y, por lo tanto, sobre el peso del vellón, como así también sobre la longitud, el diámetro promedio y la resistencia a la tracción de las fibras.

\section{Efecto de la edad}

Se encuentra bien documentado en alpacas que, a medida que aumenta la edad, se incrementa el peso del vellón (Castellaro et al., 2008; Wuliji et al., 2000; McGregor, 2006; Lupton et al., 2006) y el diámetro (Wuliji et al., 2000; McGregor y Butler, 2004; Quispe et al., 2009a). Las alpacas jóvenes producen vellones menos pesados que las adultas, por tener una menor superficie corporal (León-Velarde y Guerrero, 2001; Frank et al., 2006), sin embargo, producen vellones con fibras más finas, debido a que las esquilas tienen el efecto de incrementar el funcionamiento folicular (Rogers, 2006).

\section{Efecto del sexo}

En ganado ovino, los machos producen lanas más gruesas, así como más largas y pesadas que las hembras, pero considerando que la eficiencia de producción de lana está fuertemente relacionada con el peso vivo, independientemente del sexo, entonces la mayor producción de lana de los machos enteros, consecuencia de su mayor tamaño corporal y peso vivo, por una adecuada actividad testicular y un buen equilibrio endocrino.

Estudios realizados en alpacas demuestran que los machos producen vellones más pesados que las hembras (Castellaro et al., 1998; Wuliji et al., 2000; McGregor, 2006; Lupton et al., 2006; Montes et al., 2008, Oria et al., 2009, Quispe et al., 2008a y Quispe et al., 2009a); sin embargo, existen discrepancias sobre el efecto del sexo en el diámetro de la fibra, pues algunos investigadores como Morante et al. (2009), Quispe et al. (2009a) y Montes et al. (2008), han encontrado que los machos tienen fibras mas finas que las hembras debido a que los criadores realizan una selección de machos mucho más minuciosa e intensa que las hembras. Otros como Aylan-Parker y McGregor (2002) y Lupton et al. (2006) han reportado lo contrario, debido probablemente a que las hembras priorizan el uso de los aminoácidos ingeridos hacia la producción (preñez y lactación) en vez del abastecimiento del bulbo piloso para su excreción como fibra (Adams y Cronje, 2003). Sin embargo, Bustinza (1984), Wuliji 
et al. (2000) y McGregor y Butler (2004) consideran que no existe efecto del sexo sobre el diámetro de fibra.

\section{Efecto de la alimentación}

El crecimiento de la lana es muy sensible a los niveles de energía y de proteína ingeridos por los animales. El proceso referido del cerrado folicular (follicle shutdown) ha sido bien documentado por Schlink y Dollin (1995), y en estas circunstancias fibras sueltas pueden aparecer en los vellones. El proceso regresivo parece ser muy diferente a una normal fase telegénica (Hynd et al., 1997) y las fibras liberadas pueden ser identificadas porque sus raíces terminales son cónicas, mientras que fibras de un ciclo normal tienen terminales en forma de cepillo y los folículos suspenden su actividad para luego producir nuevas fibras en los vellones (Rogers, 2006).

En trabajos realizados en alpacas Russel y Redden (1997), Franco y San Martin (2007) y Franco et al. (2009) cuando suplementaron dietas con bajo contenido nutricional, encontraron que la producción de fibra disminuye debido a la disminución de la tasa de crecimiento y del diámetro de la fibra, observándose también que estos animales producen fibras más finas.

\section{Efecto del estado fisiológico}

La disminución en el crecimiento y en el diámetro de la lana durante la gestación y la lactación han sido reportados en ovinos en estabulación y pastoreo. Hembras gestantes y lactantes tienen fibras con menor diámetro, longitud y resistencia a la tracción, que puede mejorarse cuando se introduce en sus raciones alimentos con mayor contenido proteico (Masters y Mata, 1996).

Franco y San Martín (2007) refieren que en alpacas la gestación y la lactación causan disminución de la producción de fibra en un 17\%. También refieren que la producción de fibra disminuyó sólo en un 11\% en hembras que perdieron sus crías dentro de los 50 días post parto, y por lo tanto dejaron de lactar, sugiriendo que el efecto negativo exclusivo de la lactación sobre la producción de fibra es del 6\%. (Franco y San Martin, 2007).

Al parecer las proteínas que escapan a la degradación ruminal en ovejas durante el último tercio de gestación y la primera fase de lactación son las que incrementan el crecimiento de la lana y, en función de ello, se han planteado estrategias de alimentación por vía oral (Frey et al., 2003) en ovinos o por vía intraperitoneal en alpacas (Caso y Quispe., 2009). Esto indicaría que existe insuficiente producción de proteína microbiana durante este 
periodo, o que la composición de aminoácidos no cubre los requerimientos de las ovejas (Masters y Mata, 1996).

\section{Efecto de la sanidad.}

Una de las mayores causas de enfermedad en las alpacas son los parásitos. Las parasitosis internas pueden reducir sustancialmente el crecimiento, el diámetro y la resistencia a la tracción de la fibra. La sarna es una de las enfermedades más importantes en alpacas y además de afectar a la fibra y a su calidad, también puede causar retardo en el crecimiento y alteración de otras funciones productivas (FAO, 2005).

Por efecto de los ectoparásitos se estima que se pierden alrededor de 700.000 dólares anuales en la producción alpaquera peruana, mientras que por efecto de la sarna, se pierden alrededor de 300 mil dólares en la producción de fibra en alpacas y vicuñas (Ramos et al., 2000).

En las alpacas la mayor velocidad de crecimiento del vellón ocurre entre los meses de Noviembre y Abril y, en el mismo periodo, concurren varios factores que favorecen principalmente a la nematodiasis parasitaria, entre ellos, la época de mayor precipitacion pluvial y mejor temperatura ambiental, el relajamiento inmune periparto (RIPP) y la lactación (Núñez et al., 1994).

\section{Efecto del año}

El efecto del año en el peso del vellón y en el diámetro de las fibras han sido demostrado por numerosos autores en alpacas (Castellaro et al., 1998; Wuliji et al., 2000; McGregor 2002; Franco y San Martín 2007; Paúcar et al., 2009; Quispe et al., 2009a; Quispe et al., 2009b; Gutiérrez et al., 2009), así como en ovinos y en cabras (McGregor 1989; Zhou et al., 2003 y Saghi et al., 2007). Cuando la producción forrajera aumenta, el peso de vellón medio también aumenta (De Gea, 2007).

\section{Efecto del clima}

McGregor (1998) menciona que los factores bio-geo-físicos (fotoperiodo, sistema clima-vegetación, sistema suelo-planta, entre otros) son factores que afectan el crecimiento del cashmere y del mohair. En referencia a las alpacas y camélidos debido a que éstos se crían en un sistema extensivo con pastos naturales, el clima ejerce influencia a través de la producción forrajera sobre el crecimiento y el diámetro de la fibra, debido a la precipitación 
anual (Quispe et al., 2008a). Asimismo, en ovinos el crecimiento de la fibra a lo largo del año sufre variaciones estacionales (Naylor y Hansford, 1999).

Por otra parte, está demostrado el efecto del fotoperiodo sobre el crecimiento de la lana. Variaciones de las horas luz de los días a lo largo del año, explicarían, a través de un complejo control hormonal, aún no conocido totalmente, las variaciones en la producción de lana. Se ha señalado que el efecto del fotoperiodo sobre la producción de lana, proviene de un arcaico patrón de comportamiento de los ovinos, que pierden una vez al año su lana (Nagorcka, 1979; Pearson et al., 1996). Dicho efecto sobre el crecimiento de la fibra también fue reportado en mohair (Stapleton, 1978; McGregor, 1998) y cashmere (McDonald et al., 1987). No existen trabajos al respecto en fibra de camélidos debido probablemente a que en las zonas de mayor producción (Perú y Bolivia, principalmente) la variación de la longitud de día es pequeña.

\section{Efecto de la localización}

Una de las principales características de los ecosistemas altoandinos (donde se crían las alpacas) es su variabilidad climática, propia de todos los ecosistemas de montaña. Este factor hace común y recurrente fenómenos como las sequías, heladas, inundaciones y granizadas. Los habitantes tienen un conocimiento empírico de la realidad climática, sin embargo, en las comunidades campesinas, especialmente en los últimos 30 años, se habla frecuentemente de un cambio en los eventos climáticos (Gallardo et al., 2008)

En el caso del Perú el clima está modelado por cinco factores principales: la cordillera de los Andes, la célula anticiclónica del Pacífico sur, la corriente oceánica ecuatorial de El Niño, la corriente oceánica peruana y el anticiclón del Atlántico sur. De todos ellos, la cordillera de los Andes es especialmente determinante, con la presencia de muchos microclimas (Gallardo et al., 2008). Los diversos rebaños de camélidos se crían justamente en estos parajes con gran variabilidad respecto a su microgeografía, precipitación, sistema vegetal y suelo que serían los factores determinantes sobre el crecimiento y la calidad de la fibra, sea directa o indirectamente (Torres, 2001).

En alpacas se han encontrado efectos de la localización del rebaño sobre el PVS y la MDF de la fibra (Quispe et al., 2008a; Montes et al., 2008; Quispe et al., 2009a), lo mismo que sobre la fibra de guanacos (Bacchi et al., 2010), cashemere y mohair (McGregor, 1998). 


\section{Efecto de la condición corporal}

La condición corporal de un animal nos permite evaluar de manera rápida y sencilla mediante una apreciación táctil y visual - sus reservas corporales (grasa y músculo), que ayuda a estimar el estado nutricional del animal (Frutos et al., 1997). Se mide subjetivamente mediante una puntuación corporal y las escalas utilizadas para medir la condición corporal varían en los distintos países del mundo. Animales con baja condición corporal o que pierden condición corporal nos estarían indicando que la dieta de los mismos no ha sido cubierta o no se están satisfaciendo sus requerimientos nutricionales, en contra de animales que puedan tener o aumentar su condición corporal. Por ello, es de considerar que la condición corporal es una herramienta útil para evaluar el manejo nutricional al que ha estado sometido un hato de alpacas.

La nota de la condición corporal está basada en la palpación y observación de diferentes áreas del animal para determinar el nivel de cobertura de grasa. (Chayer R., 2005). Sin embargo, desde que fue definido y descrito de acuerdo con una puntuación de 5 puntos por palpación de la región lumbar, muchos han utilizado ésta región referencial, basado en que el lomo es la última parte del animal en crecimiento a desarrollar y a causa de ello es la última parte en donde se deposita grasa y también la primera en perderla (Frutos et al., 1997).

El estado nutricional en que se encuentra un animal es el resultado del balance entre consumo y gasto de energía. Si este balance es positivo, se acumulan reservas en forme de tejido graso; si es negativo, se consumirán reservas acumuladas con anterioridad. Podemos considerar, entonces, a las reservas corporales del animal, como una forma más de transferir energía de las épocas de mayor disponibilidad a los períodos de escasez (Chayer, 2005). Un gran número de factores afectan el estado nutricional de los animales que a través del pastoreo obtienen su principal fuente de alimento (Oregui et al., 1991). Desde su definición (Russel et al., 1969) este método ha sido usado en ovinos para acciones de manejo y muchos estudios han sido basados en determinar su relación con parámetros de producción (Oregui et al., 1991; Sanson et al., 1993) y reproducción (Gunn et al., 1979). También se ha utilizado este método en alpacas para estimar valores de hemoglobina (Silva-Sánchez et al. 2007), presencia de la hormona leptina y los valores séricos (Enciso et al., 2007).

Aunque está regularmente documentado que la producción y la finura de la fibra de alpaca están influenciados fuertemente por la alimentación, pues animales bien alimentados producen fibras más gruesas (Russel y Redden, 1997; Braga et al., 2007; Franco et al., 2009), 
existe poca información del efecto que tiene la condición corporal sobre la cantidad y la calidad de fibra, aunque como referencia conviene citar lo referido por Quispe et al., (2008a), quienes indican que bajo la condiciones extensivas de la ganadería alpaquera, la disponibilidad de pastos tiene un efecto marcado en el perfil del diámetro de fibra, pues en periodos donde existe poca disponibilidad forrajera baja la condición corporal y disminuye el diámetro de la fibra.

Por otro lado, Bustinza et al., (1985) estudiaron el crecimiento de la fibra durante el año, encontrando que la tasa de crecimiento fue mayor en diciembre y enero (inicio de lluvia y lluvia, respectivamente), donde se desarrolló el 25\% del crecimiento en longitud, y menor entre septiembre y octubre (época seca) donde ocurrió un 10\% de dicho crecimiento. Estos resultados se atribuyen fundamentalmente a la disponibilidad forrajera de la pradera.

\section{CLASIFICACIÓN DE LA FIBRA DE ALPACA.}

El Instituto Nacional de Defensa de la Competencia y de la Protección de la Propiedad Intelectual (INDECOPI) de Perú, aprueba mediante resolución las Normas Técnicas Peruanas (NTP) para la categorización en vellón y la clasificación de la fibra de alpaca.

La categorización se realiza con el vellón completo, teniendo en consideración el porcentaje de fibras superiores o inferiores (mayores o menores de $26.5 \mu \mathrm{m}$, respectivamente), la longitud, color, variedad (Huacaya o Suri), calidad de esquila y porcentaje mínimo de Baby (iguales o menores a $23 \mu \mathrm{m}$ ). La clasificación es el procedimiento en el que se rompe el vellón y se agrupa teniendo en cuenta el diámetro de fibra, longitud de mecha y color. De este modo, se pueden encontrar 04 categorías en los que se pueden seleccionar los vellones y 06 clases en que se pueden seleccionar los componentes del vellón-

De Los Ríos (2006) refería que en el Perú, el 20\% de la producción está dado por fibra Alpaca Huarizo (fibra gruesa, mayor de 29 micras), el 46\% por fibra Alpaca Medium Fleece (fibra semifina, entre 26.6 y 29), el 22 \% por fibra Alpaca Fleece (fibra fina, entre 23,1 y 26,5) y sólo el 12\% está conformado por fibra Alpaca Baby (fibra extra fina, menor de 23,1 micras), lo cual hace vislumbrar el enorme déficit en calidad. También indican que muchos de los vellones son canosos, pintados y canoso-pintados, y en muchos vellones se encuentra gran heterogeneidad en la estructura, ya que muchas fibras que lo conforman son de tipo medulado de forma continua o discontinua, lo cual desmerece la calidad del vellón. En función a estas consideraciones se puede apreciar que existe un deterioro genético de la alpaca 
principalmente en lo que se refiere a la finura de la fibra, aunque también en lo referente al peso del vellón, que podrían mejorarse si se realiza un adecuado programa de mejora genética.

\section{CONCLUSIONES}

Las características productivas y tecnológicas de la fibra de alpacas Huacayas se encuentran dentro de los requerimientos exigidos por la industria textil, pudiéndose postular que en la población de alpacas de la región de Huancavelica (Perú), existen buenos animales para la producción de fibra que pueden ser utilizados para realizar mejora genética. Asimismo, se puede descartar la creencia de que la mayor cantidad de fibra de alpacas que se produce en Huancavelica es semifina y gruesa, pues la mayor cantidad de vellones estarían clasificados como extrafina y fina, pudiendo por tanto los productores exigir mayor precio por su fibra.

\section{AGRADECIMIENTOS}

Se agrade el apoyo económico recibido a través del proyecto "Mejora de los Ingresos Económicos y Condiciones de vida de familias pobres mediante fortalecimiento de capacidades y mejora de Alpacas Huacaya en la Zona Alto-Andina de Huancavelica (Perú)”, financiado por el Gobierno de Navarra (España).

\section{REFERENCIAS BIBLIOGRAFICAS}

Adams N.R. y Cronjé P.B. 2003. A review of the biology linking fibre diameter with fleece weight, liveweight and reproduction in Merino sheep. Australian Journal of Agricultural Research, 54: 1 - 10.

Anderson S.L. 1976. The Measurement of Fibre Fineness and Length: The Present Position. J. Text. Inst., 67: 175-180.

Apomayta Z. y Gutiérrez G. 1998. Evaluación de características tecnológicas y productivas de la fibra en alpacas Huacaya esquiladas a los 12 y 17 meses de edad. Anales Científicos. UNA La Molina. Lima, Perú. 36: 35-42.

Aylan-Parker J. y McGregor B.A. 2002. Optimising sampling techniques and estimating sampling variance of fleece quality attributes in alpacas. Small Rumin. Res., 44: 53-64. 
Bacchi C.S., Lanari M.R. y von Thüngen J. 2010. Non-genetic factos affecting morphometric and fleece traits in guanaco (Lama guanicoe guanicoe) populations from Argentinean Patagonia. Small Rumin. Res. 88: 54-61..

Braga W., Leyva V. y Cochran R. 2007. The effect of altitude on alpaca (Lama pacos) fiber production Small Rumin. Res., 68: 323-328.

Brenes E.R., Madrigal K., Pérez F. y Valladares K. 2001. El Cluster de los Camélidos en Perú: Diagnóstico Competitivo y Recomendaciones Estratégicas. Instituto Centroamericano de Administración de Empresas http://www.cid.harvard.edu/archive/andes/documents/workingpapers/microfoundations/agr otech/peru/cluster_camelidos_peru.pdf. Accesado el 26 de Enero del 2012.

Bryant F.C., Florez A. y Pfister J. 1989. Sheep and alpaca productivity on high andean rangelands in Peru. J. Anim. Sci., 6:3078-3095.

Bustinza, V., 1984. Rendimiento del vellón de la Alpaca. Problemática Sur Andina $\mathrm{N}^{\circ} 7$. IIDSA - Universidad Nacional del Altiplano. Puno. Perú.

Bustinza A.V., Sapana R. y Medina G. 1985. Crecimiento de la fibra de alpaca durante el año. En. Memoria del. Proyecto Piel de Alpaca: Informe final. Univ. Nac. del Altiplano Puno Perú. p. 115-120.

Bustinza V. 2001. La alpaca, conocimiento del gran potencial andino. Puno. Univ. Nacional del Altiplano. 343 pág.

Butler K.L. y Dolling M. 1992. Calculation of the heritability of spinning fineness from phenotypic and genetic parameters of the mean and CV of fibre diameter. Aust. J. Agric. Res. 43: 1441-1446.

Butler K.L. y Dolling M. 1995. Spinning fineness of wool. J. Text. Inst. 85(1): 164-166.

Carhuapoma P., Sáenz A.P. y Quispe E.C. 2009. Efecto de la condición corporal sobre el peso de vellón y finura de fibra en alpacas Huacaya (Vicugna pacos) color blanco en la región Huancavelica. Tesis para optar el Titulo de Ing. Zootec. UNH. Huancavelica. 63 pág.

Carpio M. 1991. La fibra de camélidos. En: Novoa y Flores. Producción de rumiantes menores: Alpacas. Lima: RERUMEN. p. 297-359.

Castellaro, G., J. Garcia-Huidobro y P. Salinas. 1998. Alpaca liveweight variations and fiber production in Mediterranean range of Chile. J. Range Manage., 51: 509-513.

Caso E. y Quispe E.C. 2009. Influencia de aminoplex forte en la tasa de crecimiento mensual y producción total de fibras de alpacas hembras. Tesis para optar el Título de Ing. Zootecnista. Universidad Nacional de Huancavelica. Perú. 59 pág.

Chayer R. 2005. Condición corporal como herramienta para el seguimiento del manejo nutrición de los vientres en rodeos de cría. Universidad Nacional del Centro. Facultad de Ciencias Veterinarias. Argentina. En http://www.vet.unicen.edu.ar/edcont2007/ entornovirtual/Ano\%20I\%20General/Chayer_condicion\%20corporal.pdf. Accesado el 25 de Marzo de 2010. 
Cervantes I., Goyache F., Pérez-Cabal M.A., Nieto B., Salgado C., Burgos A. y Gutierrez J.P. 2010. Genetic parameters and relationships between fibre and type traits in two breed of Peruvian alpacas. Small Rumin. Res., 88:6-11.

Contreras A. y Quispe E.C. 2010. Estructura cuticular y características físicas de la fibra de alpaca Huacaya (Vicugna pacos) de color blanco en la Región de Huancavelica. Tesis para optar el Titulo de Ing. Zoot. Universidad Nacional de Huancavelica. 90 pág.

De Gea G. 2007. El ganado lanar en la Argentina. 2da. Edición. Universidad Nacional de Rio Cuarto. Córdova, Argentina. 245 pág.

De Los Ríos E. 2006. Producción textil de fibras de camélidos sudamericanos en el área altoandina de Bolivia, Ecuador y Perú. Organización de las Naciones Unidas para el Desarrollo Industrial (UNIDO). https://www.unido.org/filestorage/download/?file_id=58563. [Accesado el 26 de Septiembre del 2007].

De Groot G.J. 1995. The Effect of coefficient of Variation of Fibre diameter in Wool tops on Yarn and Fabric Properties. J. Text. Inst., 86(1): 164-166.

FAO. 2005. Situación Actual de los Camélidos Sudamericanos en el Perú. Organización de las Naciones Unidas para la Agricultura y la Alimentación. Proyecto de Cooperación Técnica en apoyo a la crianza y aprovechamiento de los Camélidos Sudamericanos en la Región Andina TCP/RLA/2914. En: http://www.fao.org/regional/Lamerica/prior/ segalim/animal/paises/pdf/2914per.pdf. Accesado el 30 de marzo de 2009.

Fish V.E., Mahar T.J. y Crook B.J. 1999. Fibre curvature morphometry and measurement. International Wool Textile Organization. Nice Meeting. Report N CTF 01.

Franco F. y San Martin F. 2007. Efecto del Nivel Alimenticio sobre el rendimiento y calidad de fibra en alpacas. Sistema de revisiones en Investigación Veterinaria en UNMSM. En: http://www.unmsm.edu.pe/veterinaria/files/SIRIVS\%20N1.pdf Accesado el 14 de Abril de 2010.

Franco F., San Martin F. Ara M., Olazábal L y Carcelén F. 2009. Efecto del nivel alimenticio sobre el rendimiento y calidad de fibra en alpacas. Rev. Inv. Vet. Perú. 20(2): 187-195.

Frank E.N., Hick M.V.H., Gauna C.D., Lamas H.E., Renieri C. y Antonini M. 2006. Phenotypic and genetic description of fibre traits in South American domestic camelids (llamas and alpacas). Small Rumin. Res., 61: 113-129.

Frutos P., Mantecón A.R. y Giráldez F.J. 1997. Relationship of body condition store and live weight with body composition in mature Churra ewes. Animal Science, 64: 447-452.

Galal E.S.E. 1986. Selection for increased production in multi-purpose sheep and goats. Small ruminant production in the developing countries. Proc. FAO Animal Production and Health Paper. Rome Italy. No. 58.

Gallardo M., Gómez A., Torres J. y Walter A. 2008. Cambio climático en el Perú: instituciones, investigadores, políticas, programas, proyectos y recopilación bibliográfica. Primera aproximación. ITDG. Lima Perú. 130 pág. 
Garnsworthy R.K., Gully R.L., Kandiah R.P., Kenins P., Mayfield R.J. y Westerman, R. A. 1988. Understanding the Causes of Prickle and Itch from Skin Contact of Fabrics, Australiasian Textiles 8(4): 26-29.

Gerken M. 2009. Relationships between integumental characteristics and thermoregulation in South American camelids. Animal 1-9.

Grigg G.C., Beard L.A. y Augee M.L. 2004. The evolution of endothermy and its diversity in mammals and birds. Physiological and Biochemical Zoology 77: 982-997.

Gunn R.G., Doney J.M. y Russel A.J.F. 1979. Fertility in Cheviot ewes. 2. The effect of level of pre-mating nutrition on ovulation rate and early embyo mortality in North and South Country Cheviot ewes in moderately good condition at mating.. Animal Production, 29: 17-23.

Gutiérrez J.P., Goyache F., Burgos A. y Cervantes I. 2009. Genetic análisis of six production traits in Peruvian alpacas. Livestock Science 123: 193-197.

Hack W., McGregor B., Ponzoni R., Judson G., Carmichael I. \& Hubbard D.. 1999. Australian alpaca fibre: Improving productivity and marketing. A report for the Rural Industries Research and Development Corporation. RIRDC. Australia. 164 pág.

Hansford K.A. 1996. Wool strength and topmaking. En Papers Top-Tech. Geelong, Australia. 284-292.

Hoffman E. y Fowler M.E. 1995. The Alpaca book. Clay Press Inc., Herald, California. 255 pp.

Holt C. 2006. A survey of the relationships of crimp frecuencia, micron, character y curvature de fibra. A report to the Australian Alpaca Association. Pambula Beach NSW. Australia.

Huanca T., Apaza N. y Lazo A. 2007. Evaluación del diámetro de fibra en alpacas de las comunidades de los distritos de Cojata y Santa Rosa - Puno. Arch. Latinoamer. Prod. Anim., 15(Supl. 1):480.

Hynd P.L., Ponzoni R.W. y Hill J.A. 1997. Can selection for skin traits increase the rateo $f$ genetic progress in merino breeding programs?. Proc. Assoc. Advanc. Anim. Breed. Genet., 12: 6-10.

Kosgey I.S. y Okeyo A.M. 2007 Genetic improvement of small ruminants in low-input, smallholder production systems: Technical and infrastructural issues. Small Rumin. Res., 70: 76-88.

León-Velarde C.U y Guerrero J. 2001. Improving quantity and quality of Alpaca fiber; using simulation model for breeding strategies. http://inrm.cip.cgiar.org/home/publicat/01cpb023.pdf. [Accesado el 18 de abril de 2010].

Liu X., Wang L. y Wang X. 2004. Evaluating the Softness of Animal Fibers. Textile Res. J., 74(6): 535-538.

Lupton C.J., McColl A. y Stobart R.H. 2006. Fiber characteristics of the Huacaya Alpaca. Small Rumin. Res., 64: 211-224. 
Martindale J.G. 1945. A new method of measuring the irregularity of yarns with some observations on the origin of irrgularities in worsted slivers and yarns. J. Text. Inst. 36: T35-T47.

Martínez, Z., Iñiguez, L.C. y Rodríguez, T. 1997. Influence of effects on quality traits and relationships between traits of the llama fleece. Small Rumin. Res., 24: 203-212.

Masters D. y Mata G. 1996. Responses to feeding canola meal or lupin seed to pregnant, lactating and dry ewes. Aust. J. of Agric. Res. 47:1291-1303.

Mayhua P., Quispe E.C., Montes M. y Alfonso L. 2011. Differences in fibre diamter profile between shearin periods in White-Huacaya alpacas (Vicugna pacos). En: Fibre production in South American Camelids and other fibre animals. Edity by: Perez-Cabal et al. Wageningen Academic Publishers. The Netherlands.

McDonald B.J., Hoey W.A. y Hopkins P.S. 1987. Cyclical fleece growth in cashmere goats. Aust. J. Agric. Res. 38: 597-609.

McGregor, B.A. 1989. Nutrition management to maximise cashmere production. Proceedings 3rd International Cashmere Conference, Adelaide, Australian Cashmere Growers Association.

McGregor B.A. 1998. Nutrition, management and other environmental influences on the quality and production of mahair and cashmere with particular referencia to Mediterranean and annual temperate climatic zones: A review. Small Rumin. Res. 28: 199-215.

McGregor B.A. 2002. Comparative productivity and grazing behaviour of Huacaya alpacas and peppin Merino sheep grazed on annual pastures. Small Rumin. Res., 44: 219-232.

McGregor B.A. 2006. Production attributes and relative value of alpaca fleeces in southern Australia and implications for industry development. Small Rumin. Res., 61: 93-111.

McGregor B.A. y Butler K.L. 2004. Sources of variation in fibre diameter attributes of Australian alpacas and implications for fleece evaluation and animal selection. Aust. $J$. Agric. Res., 55: 433-442.

McLennan N. $\quad$ y $\quad$ Lewer $\quad$ R. 2005. Wool production Coefficient of variation of fibre diameter (CVFD). En: http://www2.dpi.qld.gov.au/sheep/10003.html. Accesado el 25 de Marzo del 2010.

Montes M, Quicaño I., Quispe R., Quispe E.C. y Alfonso L. 2008. Quality characteristics of Huacaya Alpaca fibre produced in the Peruvian Andean Plateau region of Huancavelica. Span. J. of Agric. Res. 6(1):33-38.

Morante R., Goyache F., Burgos A., Cervantes I., Péres-Cabal M.A. y Gutiérrez J.P. 2009. Genetic improvemente for alpaca fibre production in the Peruvian Altiplano: the Pacomarca experience. Anim. Genet. Resour. Informat. 45: 37-43.

Mueller J.P. 1991. Planes de mejoramiento genético ovino en la Argentina. En: Memorias de la Jornada de actualización en Producción Ovina. Concepción del Uruguay. Comunicación Técnica PA 177. 
Nagorcka B.N. 1979. The effect of photoperiod on wool growth. En: Physiological and Environmental Limitation to Wool Growth, por: Black J.L. y Reis P.J. University of New England Publishing, Armandale, New South Wales 127-137.

Naylor G.R.S. y Stanton J. 1997. Time of shearing and the diameter characteristics of fibre ends in the processed top: An opportunity for improved skin comfort in garments. Wool Tech. Sheep Breeding, 45(4): 243-255.

Naylor G.R.S. y Hansford K.A. 1999. Fibre End Diameter Properties in Processed top Relative to the Staple for Wool Grown in a Meditarranean Climate and Shorn in Different Seasons. Wool Tech. Sheep Breeding, 42(2): 107-117.

Newman, S-A. N. y Paterson D. J.. 1994. Effect of level of nutrition and season on fibre growth in alpacas. Proc. New Zealand Soc. Anim. Product.,54: 147-150.

Núñez A., Rojas M. y Leyva V. 1994. Anematódico antes del parto y producción de fibra y peso vivo en alpacas. Investigaciones Pecuarias. 7(2).

Oregui L.M., M.S. Vicente, J. Garro y M.V. Bravo. 1991. The relationship between body condition score and body weight in Latxa ewes. Gobierno vasco, departamento de agricultura y pesca servicio de investigación y mejora agraria. Options Méditerranéennes Série Séminaires, 13: 109-112.

Oria I., Quicaño I., Quispe E. y Alfonso L. 2009. Variabilidad del color de la fibra de alpaca en la zona altoandina de Huancavelica-Perú. ITEA. Animal Genetic Resources Information, 45: 79-84.

Paúcar R., Alfonso L. y Quispe E.C. 2009. Evaluación genética de alpacas Huacaya de color blanco en Huancavelica Perú. Proc. V Congreso Mundial sobre Camélidos. Riobamba Ecuador, 1: 78.

Pearson A.J., Parry A.L., Ashby M.G., Choy V.J., Wildermoth J.E. y Craven A.J. 1996. Inhibitory effect of increased photoperiod on wool follicle growth. J. Endocrinol. 148: 157-166.

Poma A.G., Ventura C.E. y Quispe E.C. 2009. Caracterización del perfil del diámetro de fibra en alpacas Huacaya de color blanco. Tesis para optar el título de Ing, Zoot. Universidad Nacional de Huancavelica, 99 pág.

Ponzoni R.W., R.J. Grimson, J.A. Hill, D.J. Hubbard, B.A. McGregor, A. Howse, I. Carmichael y G.J. Judson. 1999. The inheritance of and association among some production traits in young Australian alpacas. En: http://www.alpacas.com/AlpacaLibrary/InheritanceTraits.aspx. Accesado el 16 de Abril de 2009.

Quispe E.C., Mueller JP., Ruiz J., Alfonso L. y Gutiérrez G. 2008a. Actualidades sobre adaptación, producción, reproducción y mejora genética en camélidos. Universidad Nacional de Huancavelica. Primera Edición. Huancavelica, Perú, pp. 93-112. 
Quispe E.C., R. Paúcar, A. Poma, D. Sacchero y J.P. Mueller. 2008b. Perfil del diámetro de fibras en alpacas. Proc. de Seminario Internacional de Biotecnología Aplicada en Camélidos Sudamericanos. Huancavelica. Perú.

Quispe E.C., Alfonso L., Flores A., Guillén H. y Ramos Y. 2009a. Bases to an improvement program of the alpacas in highland region at Huancavelica-Perú. Archivos de. Zootecnia. 58 (224): 705-716.

Quispe E.C., Rodríguez T.C., Iñiguez L.R. y Mueller J.P.. 2009b. Producción de fibra de alpaca, llama, vicuña y guanaco en Sudamérica. Animal Genetic Resources Information, 45: $1-14$.

Quispe E.C., H. Ramos H. Mayhua P. y Alfonso L. 2010. Fibre characteristics of vicuña (Vicugna vicugna mensalis). Small Rumin. Res. [In press].

Quispe E.C. 2010. Estimación del progreso genético de seis esquemas de selección en alpacas (Vicugna pacos l.) Huacaya con tres modelos de evaluación en la región altoandina de Huancavelica. Tesis para optar el Grado de Doctor. UNALM. Lima, Perú.

Ramos H., Castrejón M., Valencia N. y Sas Zevallos P. 2000. Control de sarna sarcóptica (Sarcoptes scabiei var. Aucheniae) en alpacas (Lama pacos) en Perú, con ivermectina 1\% P/P inyectable de larga acción. Tesis para optar el Título de Ing. Zootecnista. Universidad Nacional de Huancavelica. Perú. 68 pág.

Renieri C., Pacheco C., Valbonesi A., Frank E. y Antonini M. 2007. Programa de mejoramiento genético en camélidos domésticos. Arch. Latinoamer. Prod. Anim., 15: 205210.

Rodríguez, T. 2006. Producción de fibra de camélidos, calidad de fibra de llama descerdada y clasificada. Edit. Instituto de Investigaciones Agropecuarias Facultad de Agronomía, UMSA. Bolivia. 361-374.

Rogers G. 2006. Biología of the wool follicle: an excursion into a unique tissue interaction system waiting to be re-discovered. Experimental Dermatology, 15: 931-949.

Rui-wen F., Chang-sheng D., Jun-zhen Z., Xiao-yan H, Jun-ping H., Yu-hong R y Rui B. A Study on the Structure and Characteristics of the Alpaca (Lama pacos) Fibre. 2008. Act. Laser Biol. Sinic., 17(2): 224-228.

Ruiz de Castilla M. 2004. Genética y Mejoramiento de los Animales Domésticos. Edit. Universitaria Univ. Nac. San Antonio Abad del Cusco Perú. pp. 286.

Russel A.J.F., Doney J.M. y Gunn.G. 1969. Subjective assessment of body fat in live sheep. J. Agric. Sci., Camb., 72: 451-454.

Russel A.J. y Redden H.L. 1997. The effect of nutrition on fibre growth in the alpaca. Anim. Scie., 64: 509-512.

Sachero D. 2005. Utilización de medidas objetivas para determiner calidad de lanas. En: Memorias del VII Curso: Actualización en Producción Ovinas. Bariloche, Argentina. 207221. 
Safari E., Fogarty N.M. y Gilmour A.R. 2005. A review of genetic parameter estimates for wool, growth, meat and reproduction traits in sheep. Livest. Product. Sci., 92: 271-289.

Saghi D.A., Shiri S.A. y Nikbakhti M. 2007. Study on Cashmere Quality and Hair Percentage of Black Goat of Southern Khorasan for Stable Development of Agriculture. Pakistan J. Nutrition 6 (4): 397-398.

Sanson D. W., West T. R., Tatman W. R., Riley M. L., Judkinsz M. B. y Moss G. E. 1993. Relationship of Body Composition of Mature Ewes with Condition Score and Body Weight. J. Anim. Sci., 71:1112-1116.

Schlink A.C. y Dollin A.E. 1995. Abnormal shedding contributes to the reduce staple strength of tender wool in Western Australian Merinos. Wool Technol Sheep Breed. 43: 268 - 284.

Silva-Sánchez P., Mendoza G., Trahtemberg, T., Arróspide C., y Echevarría L. 2007. Variación de los valores de hemoglobina en alpacas con pobre condición corporal trasladadas desde Cerro de Pasco a Lima. APPA - Proc. ALPA, Cusco, Perú.

Stapleton D.L. 1978. Mohair production and seasonal variability in fleece of the Australian Angora goat. PhD. Thesis, University of New England, Armandale, New South Wales. Australia.

Torres J. 2001. Estrategia y plan de acción de la biodiversidad para el departamento de Huancavelica como base de su desarrollo sostenible. Comunidad Andina. Banco Interamericano de Desarrollo. Lima - Perú. 132 pág.

Torres de Jasaui J. Vélez V., Zegarra J. y Díaz G. 2007. Caracterización de la histología de la piel de alpaca. Proc. APPA - ALPA. Cuzco, Perú.

Turner H.N., Hayman R.H., Riches J.H., Roberts N.F. y Wilson L.T., 1953. Physical Definition of Sheep and Their Fleece for Breeding and Husbandry Studies. Divisional Report No. 4 (Series S.W.-2), Division of Animal Health and Production, Commonwealth Scientific and Industrial Research Organisation, Melbourne, Australia, 92 pág.

Villarroel J. 1963. Un estudio de la fibra de alpaca. Anales Científicos UNALM, 1:246-274.

Wang X., Wang L. \& Liu, X. 2003a. The Quality and Processing Performance of Alpaca Fibres: A report for the Rural Industries Research and Development Corporation. RIRDC Putlication No 03/128. Australia. 132 pág.

Wang G., Zhang W., Postle R. y Phillips D. 2003b. Evaluating Wool Shirt Comfort with Wear Trials and the Forearm Test. Tex. Res. J. 73(2): 113-119.

Wang, L., Liu, X. y Wang, X. 2004. Changes in Fibre Curvature during the Processing of Wool and Alpaca Fibres and their Blends, in College of Textiles, Donghua University. Proc. of the Textile Intitute 83rd World Conference. The Textile Institute \& Donghua University, Manchester, UK \& Shanghai, PR China 449-452.

Wang H.M., Xin L. y Wang X. 2005. Internal Structure and Pigment Granules in Coloured Alpaca Fibers. Fibers and Polymers, 6: 263-268. 
Wuliji T. 1993. Alpaca fibre production, fibre growth seasonality and fibre characteristics variation in a cool-temperate environment of New Zealand. Proc. XVII Internat. Grassland Congress 1495-1945.

Wuliji T., Davis G.H., Dodds K.G., Turner P.R., Andrews R.N. y Bruce G.D. 2000. Production performance, repeatability and heritability estimates for live weight, fleece weight and fiber characteristics of alpacas in New Zealand. Small Rumin. Res., 37: 189201.

Wurzinger M., Willam A., Delgado J., Nürnberg M., Valle Zárate A., Stemmer A., Ugarte G y Sölkner J. 2008. Design of a village breeding programme for a llama population in the High Andes of Bolivia . J. Anim. Bred. Genetic., 125:311-319. 